\title{
Why industrial production of red blood cells from stem cells is essential for tomorrow's blood transfusion
}

\author{
Luc Douay*,1,2 \\ ${ }^{1}$ Erypharm, 27 Rue Chaligny, Paris 75012, France \\ ${ }^{2}$ Sorbonne Université, Centre de recherche Saint Antoine, 27 Rue Chaligny, Paris 75012, France \\ *Author for correspondence: luc.douay@aphp.fr
}

"The requirement for the availability of blood products adapted to the needs of all is a permanent public health challenge"

First draft submitted: 12 March 2018; Accepted for publication: 31 July 2018; Published online: 24 September 2018

Keywords: adult stem cells $\bullet$ blood supply $\bullet$ cultured red blood cells $\bullet$ erythrocyte $\bullet$ hematopoietic stem cells $\bullet$ immortalized erythroid progenitor lines $\bullet$ large-scale production $\bullet$ transfusion

Blood transfusion remains the only symptomatic treatment for many emergencies and chronic diseases of red blood cells (RBCs), such as sickle cell anemia, myelodysplasias, leukemias and some other cancers. Some patients are transfused all their lives and receive more than 1000 units of packed RBCs (UPR) through their lifetime. However, the more a patient is transfused, the more it increases the risk of immunization against the allogenic RBCs, frequently leading to transfusion-impasses with potentially fatal complications.

The requirement for the availability of blood products adapted to the needs of all is a permanent public health challenge. Despite the considerable progress made, transfused patients are still exposed to certain residual risks which justify, in our countries today, important expenditure for the safety of each donation. Public health costs are enormous which include the prescription of UPR, repeated hospitalizations in special care units and the social cost of disability.

\section{Present \& future challenges of blood transfusion}

Of the 112.5 million blood donations collected globally each year, approximately half of these are collected in high-income countries, home to $19 \%$ of the world's population [1]. Of course, the requirements vary considerably between different countries. In developed countries, the problem is both that of transfusion impasses due to secondary hyperimmunization with polytransfusion, and the aging of the population which has two important consequences [2]. First, it leads to a considerable development of blood cancers whose incidence increases with age and which have important transfusion needs. By 2050, their incidence will increase by $60 \%$ in France and by $100 \%$ in the USA. Second, the number of individuals within the age range able to donate blood will become much less than is required to meet the need of blood products. While the general population will increase by $16 \%$ in France and $49 \%$ in the USA, the population of blood-giving age is only 6 and 35\%, respectively [3]. In developing countries, the problem is not only that of supply but also of infectious security by inadequate biological detection of pathogens. In all countries, health crisis situations may impair the collection of blood, such as outbreaks of Ebola in Africa and Chikungunya or Dengue in the Caribbean/Guyana.

\section{Rethinking the blood supply model}

An ideal transfusion would allow: quantitative self-sufficiency on a global scale; qualitative self-sufficiency by producing $100 \%$ compatible receiver blood and thus avoid any risk of immunization; protection against infectious health risks, freeing themselves from the donor source; and eliminating metabolic complications, including iron overload as a result of post-transfusion hemolysis. 
The in vitro generation of RBCs is now possible. The concept of cultured RBCs (cRBCs) emerged, which responds to the present and future challenges of blood transfusion. Going forward, it is now a matter of designing large-scale production technology to allow the generation of cRBCs from stem cells, in a similar manner to the mass production of a drug. This approach is emblematic of the problems that regenerative medicine will have to overcome for all its indications, namely the design of large-scale bio-engineering tools to be able to carry out clinical trials.

The stem cell revolution potentially opens the way to blood for everyone by making it quantitatively sufficient, permanently available and without any risk of transmission of infectious agents. We can indeed now produce in vitro large quantities of cRBCs from several types of human stem cells. The concept of $\mathrm{cRBC}$ is therefore a reality. All upstream research steps have been successfully completed, including the demonstration of the feasibility of human injection [4].

\section{To date three key types of stem cell are potential sources of cRBCs} Hematopoietic stem cells from blood or umbilical cord

Easily accessible, hematopoietic stem cells (HSC) have been widely used worldwide for over 30 years as an alternative to bone marrow transplantation for the treatment of blood diseases. The technologies of collection, preparation and freezing are well controlled. HSCs present in the blood (so-called peripheral blood stem cells) are readily accessible. HSCs are mobilized through the administration of G-CSF to the donor which facilitates the temporary release of these cells from the bone marrow and are then easily obtained from the blood. HSCs are also obtained from cord blood. An international network of cord blood banks including nearly 1 million units is available [5]. This is a source that could be useful for the selection of the main erythrocyte phenotypes (ABO, Rh, Kell). HSCs have many strengths: natives, therefore not genetically modified, they pose no problem of acceptance by regulatory agencies; having a large capacity for proliferation and differentiation, meaning the equivalent of several hundreds of UPR could be generated from a single mobilized blood sample [6]. Their main limitation is batch production and donor dependence.

\section{Induced pluripotent stem cells}

Invented in 2007 by Yamanaka [7], induced pluripotent stem cells (iPSCs), obtained by the reprogramming of any type of differentiated adult cell, possess the characteristics of pluripotent stem cells (e.g., embryonic stem cells [ESCs]), in other words, unlimited capacity for in vitro proliferation and the ability to differentiate between any cell type of the body. IPSCs have numerous advantages over ESCs: the destruction of embryos in not required, meaning fewer ethical issues arise; they do not suffer from the extremely limited choice of donors as in ESCs; they can be obtained from carefully selected voluntary donors. IPSCs are therefore of interest for the potential production of an unlimited number of UPR for blood transfusion [8]. However, if their differentiation is controlled, the enhancement of their proliferation capacity remains a challenge that numerous teams around the world are trying to overcome. Moreover, they are genetically modified organisms that will have to meet the many requirements of the health regulatory agencies around the world. The industrial production of secure IPSCs may therefore require a few more years of research.

\section{Immortalized erythroid progenitor lines}

A more recent alternative being explored is to generate immortalized erythroid progenitor lines. The aim is to genetically modify human stem cells so that they proliferate indefinitely in the undifferentiated state and can, on demand, be induced to differentiate into RBCs [9]. This offers the potential to provide an unlimited source of stem cells for cRBC production, with all the advantages of IPSCs but with significantly higher expected proliferation and maturation capabilities. It should be noted however, these lines are also genetically modified organisms that will be subject to the same requirements of regulatory agencies as for IPSCs.

\section{The expected virtues of cRBCs}

By their very conception, one is entitled to expect real benefits from these cRBC populations in comparison to the classical transfusion. In particular, it has been established that the life span of a cRBC population is greater than that of a native RBC population [4]. This is due to the homogeneous nature of a young CRBC population compared to a population of native RBCs that is heterogeneous in age. In the context of a future clinical application, this observation is major since it predicts a significantly higher transfusion efficiency for this new blood product, 
and therefore a decrease in the number of required transfusions and consequently lower costs of patient care. An improved transfusion efficacy can therefore be expected which would make it a major asset.

There are more than 300 different antigens belonging to 37 different blood groups on the red cell membrane. The mandatory compatibility between donor and recipient is for three antigens ( $\mathrm{A}, \mathrm{B}$ and Rh1) belonging to two blood groups (ABO and Rh). In a few countries, such as France, the compatibility is extended to five additional antigens (Rh2, Rh3, Rh4, Rh5 and K1) belonging to the Rh and Kell blood groups for $80 \%$ of the patients. In these conditions, $2-3 \%$ of the total patients transfused develop antibodies against one or several blood group antigens [10]. The proportion of patients developing these antibodies increases with the number of transfusion received [11], and in some chronically transfused patient populations such as sickle cell anemia, alloimmunization against red cell antigens may occur in $20-40 \%$ of patients, leading in some cases to a transfusion impasse [12]. With the ability to select stem cell donors with red cell phenotypes of interest, cRBCs may be used to transfuse alloimmunized patients. It has been shown that using one single donor selected for the peculiarity of his erythrocyte phenotype (of the ten most immunogenic systems), it would be possible to be compatible with $95 \%$ of alloimmunized patients, and that ten donors would cover $99 \%$ of alloimmunized patients, the remaining cases corresponding to the so-called 'rare' blood groups [2]. We are therefore touching on the long awaited concept of 'universal RBCs' since the discovery of the first blood group in 1900 .

This universal donor concept is indifferently applicable to HSCs, IPSCs and immortalized lines. Donor selection on the main blood group systems will respond to transfusion impasses and ultimately avoid the risk of alloimmunization in the populations concerned. The reduction in the risks of alloimmunization is therefore another major advantage expected.

The transmission of known or emerging infectious agents is a constant concern of blood transfusion. By limiting the number of different donors, the risk of transmitting infectious agents will be greatly reduced. The persistence of known (e.g., HIV, Hepatitis C virus [HCV], etc.) and emerging (Arbovirus, E hepatitis virus, etc.) risks has a significant impact on public health. In 2005-2007, during the outbreak of Chikungunya on the island of Réunion, blood collection was stopped to limit this spread. One can therefore anticipate a significant reduction in infectious risks with the emergence of cRBCs.

It can be expected that the permanent availability of cRBC UPR will also allow a significant reduction in logistical constraints. Indeed, irrespective of age considerations, $10 \%$ of the candidates for donation are now challenged, $4 \%$ of which are for reasons of infectious risk [13]. Thus, the use of cRBCs would make it possible to simplify the recruitment of new donors to the extreme and to alleviate potential shortages. In addition, the constant availability of stem cell sources would alleviate the problem of blood shortages.

This technological revolution will not consist of a simple transfer of activity from the traditional transfusion centers to these new RBC factories, but will likely be a complementary activity, creating new jobs for specialized technicians who will: pilot new industrial devices; ensure the maintenance of equipment; manage the storage and preparation of secondary products entering the production chain; and ensure the traceability of raw materials under the control of engineers, officials and managers trained in the new manufacturing processes of biological medicines.

\section{Prospective vision of the indications of CRBCs}

In low-income countries, most blood transfusions are performed for children under 5 years of age. The indications are preferentially concerned with the management of: treatment of complications during pregnancy or childbirth; severe anemia in children; congenital hematologic disorders (e.g., sickle cell anemia, thalassemia, etc.); and trauma. In high-income countries, most transfusions are for adults over 65 years of age and indications are preferentially related to the management of hematologic disorders and trauma care [1].

By simply placing itself in a niche market, it is possible to identify indications that can benefit immediately from cRBCs.

\section{Rare blood donors}

A rare blood group is characterized by the absence of a common antigen at the surface of RBCs. The subjects concerned will develop antibodies against these antigens after a first stimulation by pregnancy or transfusion. Any individual with a rare blood group must therefore receive identical UPR, preserved frozen in a few 'blood banks of rare phenotypes'. Unfortunately, the low reserves do not allow the demand to be met in a quantitative and qualitatively satisfactory manner in most cases [14]. These situations of transfusion impasses engage the vital prognosis. In general, when a subject with a rare blood group is identified, a family study is carried out to look for 
other subjects with the same characteristic that are likely to donate their blood. It would therefore be beneficial to prepare cRBCs from a selection of these identified individuals.

\section{Hereditary diseases of RBC}

Some rare characteristics are found only in Afro-Caribbean populations. This problem is all the more important because these are populations in which frequent transfusions are necessary throughout life, especially in cases of sickle cell anemia. The situation is medically worrying in most countries (more than 50 million people with sickle cell disease worldwide and about 300,000 births per year [15]). It is economically worrying in the United States with 50,000 patients supported. In Italy and Greece, $40 \%$ of transfusions are in patients with thalassemia, another type of hereditary disease of RBCs where the problem is the same. A patient of this type receives an average of 13 UPR per year [16]. Demand is therefore high and stocks are low. Some donors have blood whose characteristics (seven to eight different phenotypes) would respond to nearly $100 \%$ of the situations.

\section{Potential future market expansion}

\section{A curative approach}

Immunization phenomena affects $1-3 \%$ of transfused patients [17]. These patients are in a potentially fatal transfusion situation. Considering that the median number of transfusions per patient is 5 UPR in Europe/USACanada/Japan/Australia/New-Zealand, more than 200,000 patients are affected every year in these countries [18]. Thanks to the cRBCs, these patients could be offered a personalized therapy.

\section{A preventative approach}

Some patients are at high risk of immunization: hematology-oncology patients and candidates for a HSC transplant; those with congenital hemoglobinopathy (thalassemia, sickle cell anemia); women of childbearing age. The transfusion of the pregnant woman is carried out with UPR compatible for $\mathrm{Rh}$, Kell phenotype to prevent immunization. In addition, it is necessary to pay particular attention to limiting the infectious risk by cytomegalovirus in seronegative women. The population concerned represents $10 \%$ of the transfused. These patients are also required to benefit from transfusions of UPR phenotyped for minor blood groups (Kell, Duffy, Kidd and Ss) for the prevention of immunization. The use of cRBCs obtained from a limited number of individuals with a red cell phenotype of interest can provide an efficient means to prevent alloimmunization in these at-risk patients' populations.

\section{Cost of cRBCs}

The annual cost of blood transfusion is becoming increasingly significant due to the legitimate demands of public health $[14,19,20]$. The market price of UPR varies considerably depending on the country (whether or not there is a network of collection of free or paid donations of blood and other forms of supply), the blood group considered (a rare blood UPR can reach up to US $\$ 5000$ or even more, excluding transport and the emergency situation or not in which the transfusion is to be carried out). The analysis of the niche markets targeted for applications of cRBCs from the proposed technology shows that, given the therapeutic benefits provided, an average price of less than 5,000 $€ / \$$ per UPR would remain competitive [21]. The gigantic potential market for $\mathrm{CRBC}$ mobilizes numerous research groups and private companies throughout the world. Undoubtedly substantial progress for industrial production are expected soon. Indeed, the development of such a new labile blood product, at an economically feasible level, would resolve almost all the current problems of blood transfusion.

\section{Conclusion}

It has been more than half a century since the search for an artificial substitute for RBCs began. To date, this has been somewhat unsuccessful and no alternative has emerged in the short term [22]. The lesson that can be learned is that it is apparently impossible to replace nature in this area as blood is such a complex tissue. The emergence of stem cells offers a new approach, in other words, to imitate nature. Our knowledge has continued to advance since the discovery of HSCs, with the discovery of ESCs, invention of IPSCs and now the production of immortalized lines, all of which have reinforced interest in this area of research. Fundamental research, even if it is never completed, has established the main bases of this approach. The time to take action is now, that is, through industrial production so that we can finally carry out the clinical trials. The goal should be to design an industrial technology that can be used to generate cRBCs, as seen in mass drug production. It will be necessary to implement technology on an industrial production prototype under pharmaceutical conditions to produce cRBCs 
concentrates to carry out clinical trials and then promote this concept for industrial development. This approach is emblematic of the problems that regenerative medicine will have to overcome for all its indications, namely the design of large-scale bioengineering tools to be able to carry out clinical trials that demonstrate the superiority of these new approaches. Hopefully, this will be a daily reality within the next few years.

\section{Translational perspective}

In the future, regenerative medicine has a number of key challenges to face: first, a scientific one, since generating cell populations with therapeutic capacity involves reproducing fundamental physiological mechanisms of the human body in vitro. The concept is made more complex by the importance of identifying the best source of stem cells to achieve both a qualitative goal, in obtaining functional cells and a quantitative goal, in achieving amplification levels compatible with a clinical application. This is made even more difficult by the fact that it seems a new source of stem cells emerges every 10 years! Fundamental science goes faster than industrial development, which is a problem common to all regenerative medicine applications - all runways must be exploited without any exclusiveness or bias. The issue of autologous versus allogeneic will also soon arise. The future, in almost any area, is certainly allogeneic development, since for logistical and regulatory reasons, it will be impossible to make biological products for every individual patient on a large scale; a second challenge is technological, from the generation of a few cells in the laboratory to the industrial production of millions or even billions of cells. We must propose revolutionary approaches and bring together scientific skills that are not normally used together such as biology, mathematics, physics, fluidics, among others. The need to design automated production tools is common to all developments in regenerative medicine. The technology will need to be simple and reproducible in order to generate cellular products that are authorized by regulatory agencies around the world. Another challenge will be that of public health. The point is to demonstrate that the medical effect rendered by these cellular therapies will be greater and less costly than current therapies. It is a public health issue because the population is aging. The goal may not be to live old but to live better. The field will then position itself as a major player in the public health of tomorrow.

\section{Financial \& competing interests disclosure}

The author is the President and scientific director of ERYPHARM, a company specialized in the production of RBCs from stem cells. The author has no other relevant affiliations or financial involvement with any organization or entity with a financial interest in or financial conflict with the subject matter or materials discussed in the manuscript apart from those disclosed.

No writing assistance was utilized in the production of this manuscript.

\section{References}

1. WHO. Blood safety and availability. Global status report on blood safety and availability (2016). www.who.int/news-room/fact-sheets/detail/blood-safety-and-availability

2. Peyrard T, Bardiaux L, Krause C et al. Banking of pluripotent adult stem cells as an unlimited source for red blood cell production: potential applications for alloimmunized patients and rare blood types. Transfus. Med. Rev. 25(3), 206-216 (2011).

3. American Association of Blood Banks. Blood FAQ (2017). www.aabb.org/tm/Pages/bloodfaq.aspx

4. Giarratana MC, Rouard H, Dumont A et al. Proof of principle for transfusion of in vitro generated red blood cells. Blood 118, 5071-5079 (2011).

5. World Marrow Donor Association. Activities report. https://wmda.info/wp-content/uploads/2017/09/20170523-WOFF-activities-report-2016.pdf

6. Giarratana MC, Kobari L, Lapillonne $\mathrm{H}$ et al. Ex vivo generation of fully mature human red blood cells from hematopoietic stem cells. Nat. Biotechnol. 23(1), 69-74 (2005).

7. Takahashi K, Tanabe K, Ohnuki M et al. Induction of pluripotent stem cells from adult human fibroblasts by defined factors. Cell 131, 861-872 (2007).

8. Lapillonne H, Kobari L, Mazurier C et al. Red blood cell generation from human induced pluripotent stem cells: perspectives for transfusion medicine. Haematologica 95(10), 1651-1659 (2010).

9. Trakarnsanga K, Griffiths RE, Wilson MC et al. An immortalized adult human erythroid line facilitates sustainable and scalable generation of functional red cells. Nat. Commun. 14(8), 14750 (2017).

10. Hoeltge GA, Domen RE, Rybicki LA et al. Multiple red cell transfusions and alloimmunization. Experience with 6996 antibodies detected in a total of 159,262 patients from 1985 to 1993. Arch. Pathol. Lab. Med. 119(1), 42-45 (1995).

11. Zalpuri S, Zwaginga JJ, le Cessie S et al. Red-blood-cell alloimmunization and number of red-blood-cell transfusions. Vox Sang. 102(2), 144-149 (2012). 
12. Rosse WF, Gallagher D, Kinney TR et al. Transfusion and alloimmunization in sickle cell disease. The cooperative study of sickle cell disease. Blood 76(7), 1431-1437 (1990).

13. Shah A, Stanworth SJ, McKechnie S. Evidence and triggers for transfusion of blood and blood products. Anesthesia 70(Suppl. 1), 10-19 (2015).

14. Fillet AM, Desmarets M, Assari S et al. Blood products use in France: a nationwide cross-sectional survey. Transfusion 56(12), 3033-3041 (2016).

15. American Society of hematology. State of sickle cell disease 2016 report. www.scdcoalition.org/pdfs/ASH\%20State\%20of\%20Sickle\%20Cell\%20Disease\%202016\%20Report.pdf

16. Piel FB, Hay SI, Gupta S et al. Global burden of sickle cell anaemia in children under five, 2010-2050: modelling based on demographics, excess mortality, and interventions. PLoS MED. 10(7), 1001484 (2013).

17. Janssen MP, van Hoeven LR, Rautmann G. European Directorate for the Quality of Medicines and Health Care of the Council of Europe (EDQM). Trends and observations on the collection, testing and use of blood components in europe: 2001-2011 report (2015) www.edqm.eu/sites/default/files/trends_and_observations_on_the_collection_testing_and_use_of_blood_and_blood_components_in_europ e_20.pdf

18. Agence nationale de sécurité du médicament et des produits de santé. Rapport d'activité hémovigilance 2015 (2016). http://ansm.sante.fr/var/ansm_site/storage/original/application/27ce3d0739821882c0cd87041b8050a7.pdf

19. Varney SJ, Guest F. The annual cost of blood transfusions in the UK. Transfus. Med. 13(4), 205-218 (2003).

20. Mercier Ythier J. Cultured blood versus donated blood: long-run perspectives of the economy of blood. Biomed. Mater. Eng. 25(Suppl. 1), 199-209 (2015).

21. Rousseau GF, Giarratana MC, Douay L. Large-scale production of red blood cells from stem cells: what are the technical challenges ahead? Biotechnol. J. 9(1), 28-38 (2014).

22. Natanson C, Kern SJ, Lurie P et al. Cell-free hemoglobin-based blood substitutes and risk of myocardial infarction and death: a meta-analysis. JAMA 299(19), 2304-2312 (2008). 